\title{
Nobel Lecture: The dawn of x-ray astronomy*
}

\author{
Riccardo Giacconi \\ Associated Universities, Inc., 1400 16th Street NW, Suite 730, \\ Washington, D.C. 20036, USA \\ and Johns Hopkins University, Baltimore, Maryland 21218, USA
}

(Published 8 August 2003)

\subsection{INTRODUCTION}

The development of rockets and satellites capable of carrying instruments outside the absorbing layers of the Earth's atmosphere has made possible the observation of celestial objects in the x-ray range of wavelength.

$\mathrm{X}$ rays of energy greater than several hundreds of electron volts can penetrate the interstellar gas over distances comparable to the size of our own galaxy, with greater or lesser absorption depending on the direction of the line of sight. At energies of a few kilovolts, $\mathrm{x}$ rays can penetrate the entire column of galactic gas and in fact can reach us from distances comparable to the radius of the universe.

The possibility of studying celestial objects in $\mathrm{x}$ rays has had a profound significance for all astronomy. Over the $\mathrm{x}$-ray to gamma-ray range of energies, $\mathrm{x}$ rays are, by number of photons, the most abundant flux of radiation that can reveal to us the existence of high-energy events in the cosmos. By high-energy events I mean events in which the total energy expended is extremely high (supernova explosions, emissions by active galactic nuclei, etc.) or in which the energy acquired per nucleon or the temperature of the matter involved is extremely high (infall onto collapsed objects, high-temperature plasmas, interaction of relativistic electrons with magnetic or photon fields).

From its beginning in 1962 until today, the instrumentation for x-ray astronomical observations has improved in sensitivity by more than nine orders of magnitude, comparable to the entire improvement from the capability of the naked eye to those of the current generation of 8- or 10-meter telescopes. All categories of celestial objects, from planets to normal stars, from ordinary galaxies to quasars, from small groups of galaxies to the furthest known clusters, have been observed. As a result of these studies it has become apparent that high-energy phenomena play a fundamental role in the formation and in the chemical and dynamical evolution of structures on all scales. X-ray observations have proved of crucial importance in discovering important aspects of these phenomena. It was from x-ray observations that we obtained the first evidence for gravitational energy release due to infall of matter onto a collapsed object such as a neutron star or black hole. It was the x-ray

TABLE I. Estimates of fluxes from sources outside the solar system. From Giacconi, Clark, and Rossi, 1960.

\begin{tabular}{|c|c|c|c|}
\hline Source & $\begin{array}{c}\text { Maximum } \\
\text { Wavelength }\end{array}$ & Mechanism for emission & Estimated Flux \\
\hline Sun & $<20 \AA$ & Coronal emission & $\sim 10^{6} \mathrm{~cm}^{-2} \mathrm{~s}^{-1}$ \\
\hline Sun at 8 light years & $<20 \AA$ & Coronal emission & $2.5 \times 10^{-4} \mathrm{~cm}^{-2} \mathrm{~s}^{-1}$ \\
\hline Sirius if $L_{X} \sim L_{\mathrm{OPT}}$ & $<20 \AA$ & $\begin{array}{l}? \\
\text { No convective zone }\end{array}$ & $0.25 \mathrm{~cm}^{-2} \mathrm{~s}^{-1}$ \\
\hline Flare stars & $<20 \AA$ & Sunlike flare? & $?$ \\
\hline Peculiar $A$ stars & $<20 \AA$ & $\begin{array}{l}\mathrm{B} \sim 10^{4} \text { gauss } \\
\text { Large } \mathrm{B} \\
\text { Particle acceleration }\end{array}$ & $?$ \\
\hline Crab nebula & $<25 \AA$ & $\begin{array}{l}\text { Synchrotron } \\
\mathrm{E}_{\mathrm{E}} \geqslant 10^{13} \mathrm{eV} \text { in } \mathrm{B}=10^{-4} \text { gauss } \\
\text { Lifetimes? }\end{array}$ & $?$ \\
\hline Moon & $<23 \AA$ & Fluorescence & $0.4 \mathrm{~cm}^{-2} \mathrm{~s}^{-1}$ \\
\hline Moon & $\sim 20 \AA$ & $\begin{array}{l}\text { Impact from solar wind } \\
\text { Electrons } \\
\phi_{\varepsilon}=0-10^{13} \mathrm{~cm}^{-2} \mathrm{~s}^{-1}\end{array}$ & $0-1.6 \times 10^{3} \mathrm{~cm}^{-2} \mathrm{~s}^{-1}$ \\
\hline SCO X-1 & $2-8 \AA$ & $?$ & $28 \pm 1.2 \mathrm{~cm}^{-2} \mathrm{~s}^{-1}$ \\
\hline
\end{tabular}

*The 2002 Nobel Prize in Physics was shared by Raymond Davis, Jr., Riccardo Giacconi, and Masatoshi Koshiba. This lecture is the text of Professor Giacconi's address on the occasion of the award. 


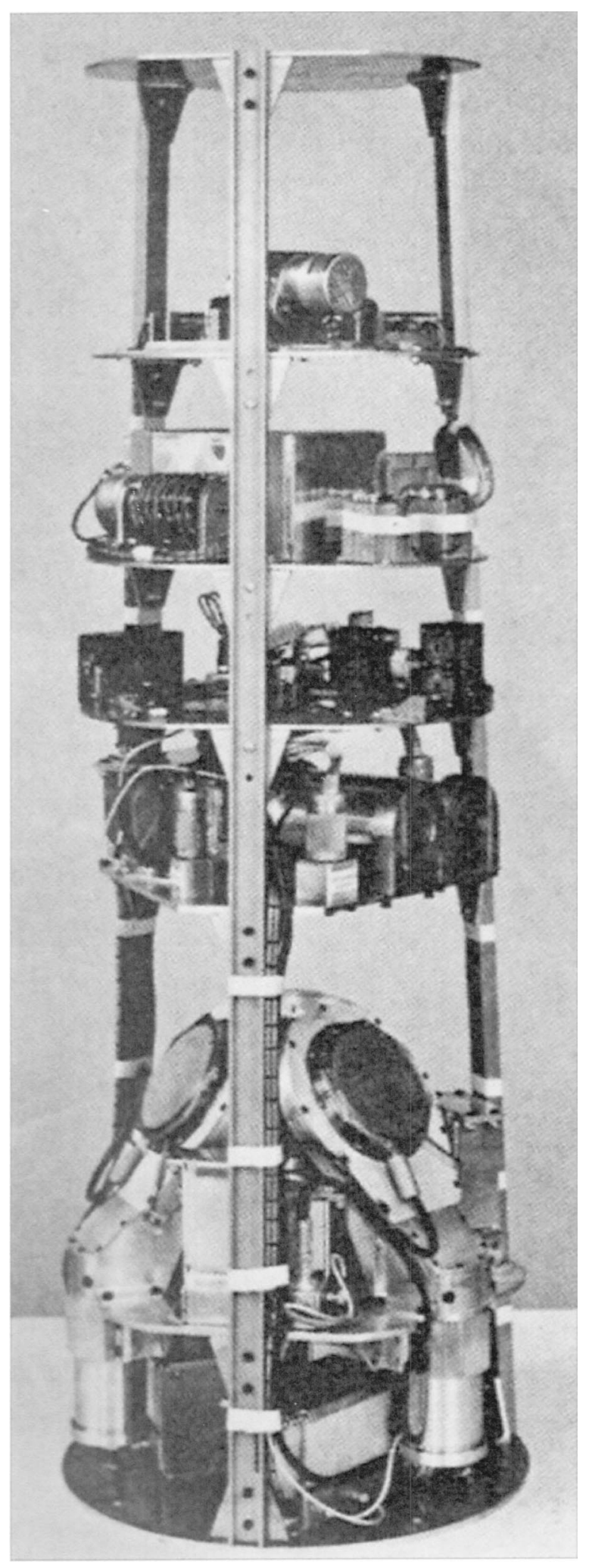

FIG. 1. The payload of the June 12, 1962 AS\&E rocket. From Giacconi and Gursky, 1974, p. 9.

emission from the high-temperature plasmas in clusters of galaxies that revealed this high-temperature component of the Universe, which more than doubled the amount of "visible" matter (baryons) present in clusters.

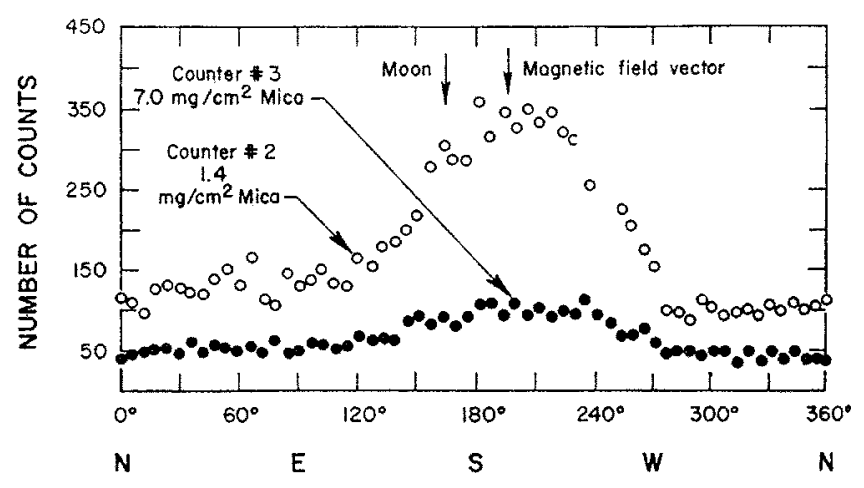

FIG. 2. The first observation of Sco X-1 and of the X-ray background in the June 12, 1962, flight. From Giacconi, Gursky, Paolini, and Rossi, 1962.

The prospects for future studies of the Universe in $\mathrm{x}$ rays are equally bright. The advent of new and even more powerful experimental techniques, such as nondispersive high-resolution spectroscopy and x-ray telescopes capable of focusing increasingly higher energies over wider fields, ensures a wide opportunity for new astronomical discoveries.

\subsection{THE BEGINNING OF X-RAY ASTRONOMY}

There had been solar x-ray observations for about 10 years by the Naval Research Laboratory (NRL) group led by Herbert Friedman and several failed attempts to find x-ray emissions from stellar objects (Hirsh, 1979) when a group at AS\&E (a small private research corporation in Cambridge, Massachusetts) started work in 1959 to investigate the theoretical and experimental possibilities for carrying out x-ray astronomy. Giacconi,

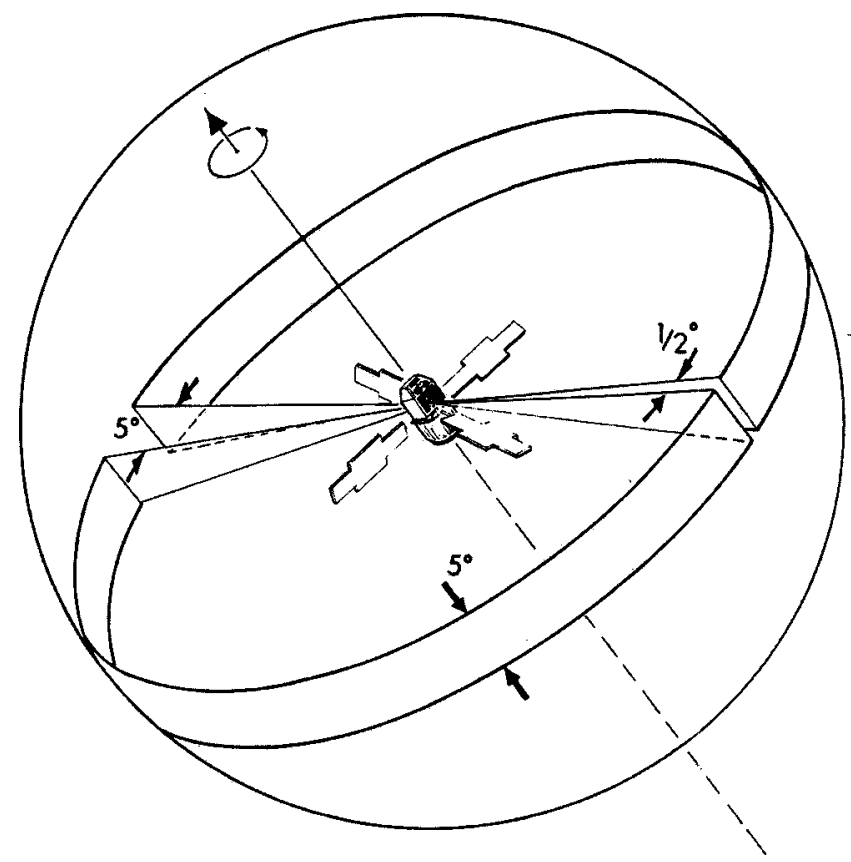

FIG. 3. The fields of view of the detectors on the UHURU satellite. From Gursky, 1970. Reprinted in Giacconi and Gursky, 1974, p. 95. 


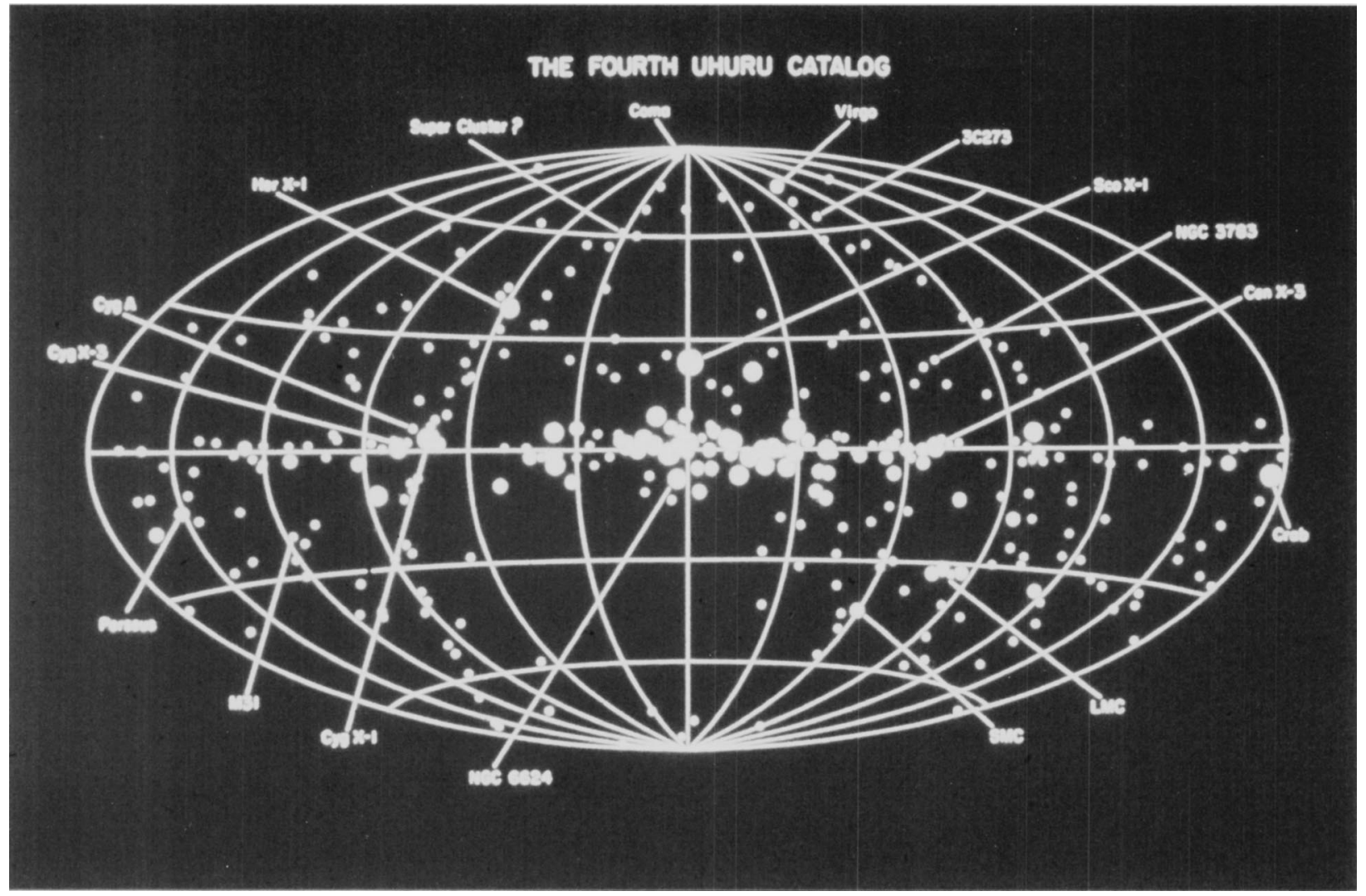

FIG. 4. The x-ray sources observed by $U H U R U$ plotted in galactic coordinates. The size of the dot is proportional to intensity on a logarithmic time scale. From Giacconi and Gursky, 1974, p. 156.

\section{CENTAURUS X-3 (2ASE $1119-60$ ) \\ May 7, 1971}

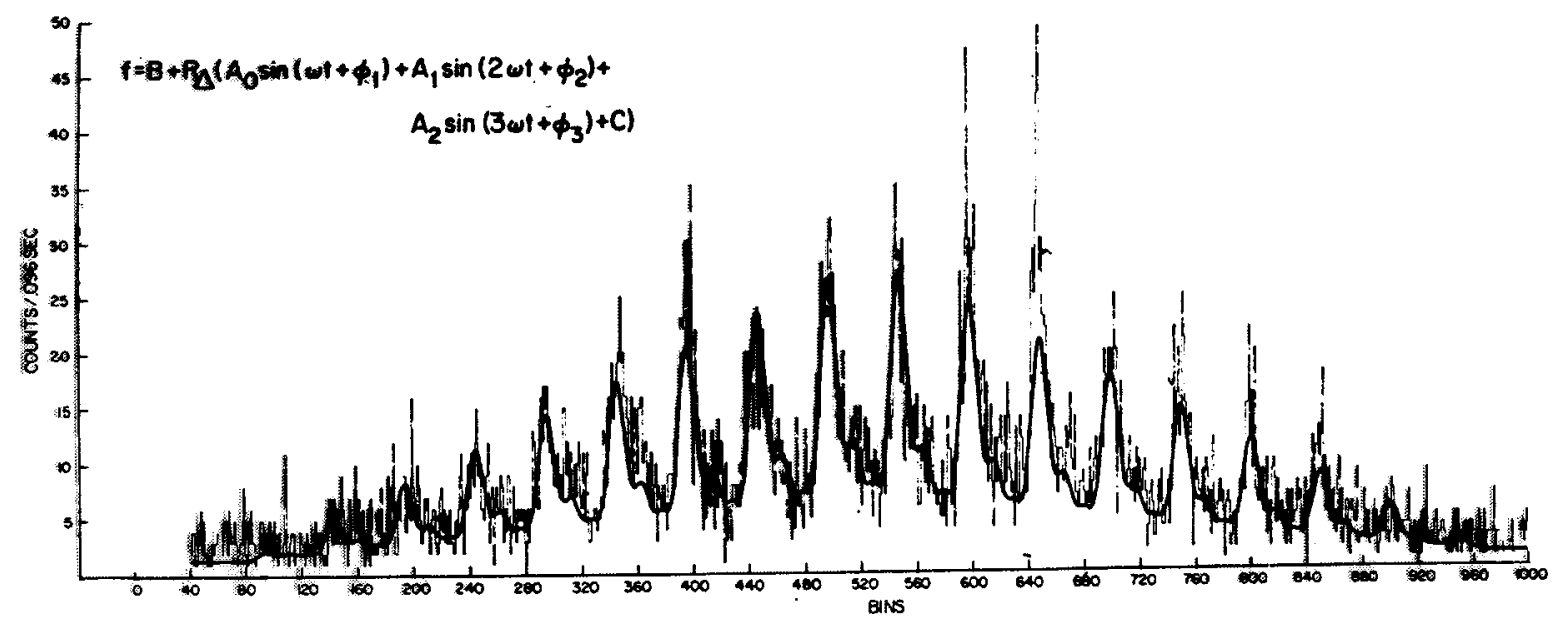

FIG. 5. X-ray pulsations of Cen X-3. From Giacconi, 1974. 


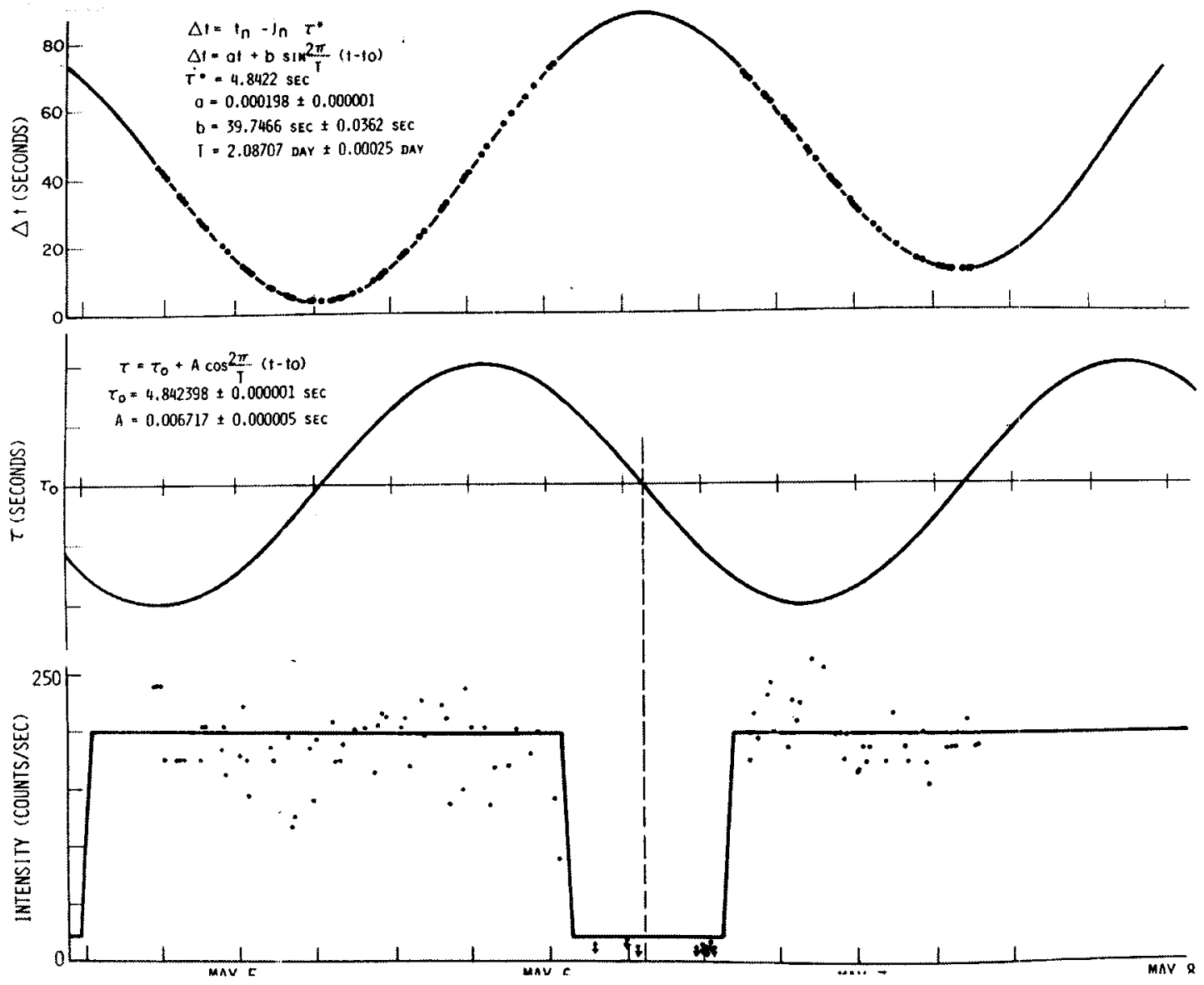

FIG. 6. Period variations and occulations of Cen X-3. From Giacconi, 1974.

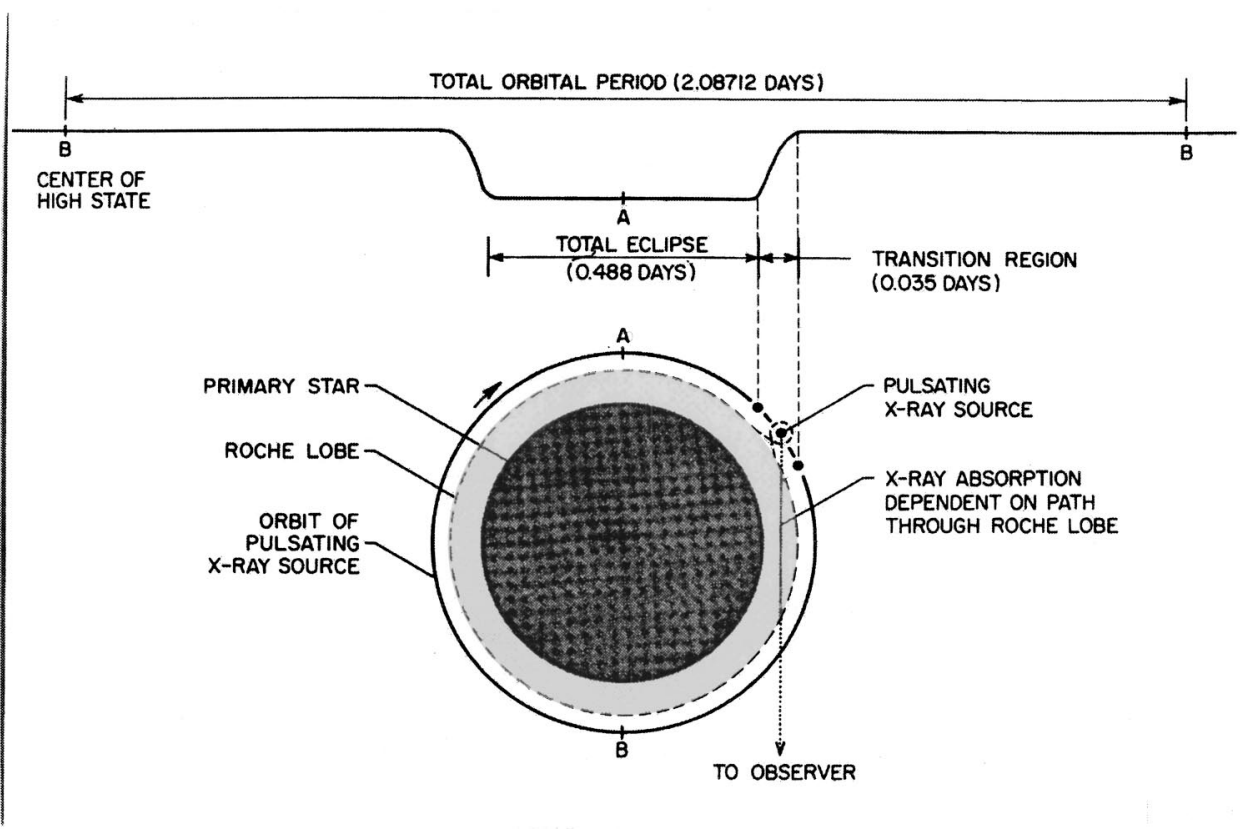

FIG. 7. A model of the Cen $\mathrm{X}-3$ binary system. Illustration of R. Giacconi. 


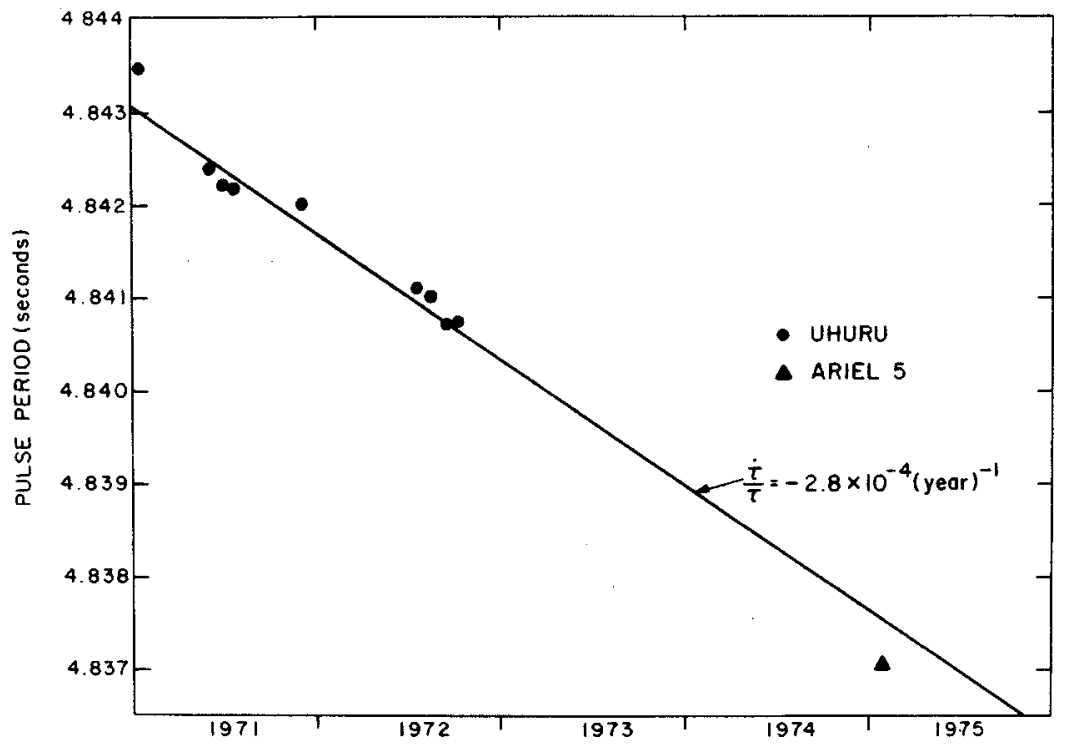

FIG. 8. Annual change of Cen X-3 pulsation period. Illustration of R. Giacconi.

Clark, and Rossi (Giacconi et al., 1960) published a document, "A Brief Review of Experimental and Theoretical Progress in X-ray Astronomy," in which we attempted to estimate expected x-ray fluxes from several celestial sources.

The results are summarized in Table I. The Sun produced $10^{6} \mathrm{x}$-ray photons $\mathrm{cm}^{-2} \mathrm{~s}^{-1}$ at Earth which could easily be detected with the then-available counters with sensitivities of about $10-10^{2}$ photons $\mathrm{cm}^{-2} \mathrm{~s}^{-1}$. But if all the stars emitted $\mathrm{x}$ rays at the same rate as the Sun, we would expect fluxes at earth as small as $10^{-4}$ photons $\mathrm{cm}^{-2} \mathrm{~s}^{-1}$. Other possible sources, such as supernova remnants, flare stars, peculiar A stars, etc., were considered, and great uncertainty had to be assigned to the estimates of their x-ray fluxes. It seemed that the brightest source in the night sky could be the Moon, due to fluorescent emission of lunar material under illumination of solar $\mathrm{x}$ rays.

We designed an experiment capable of detecting $0.1-1$ photon $/ \mathrm{cm}^{-2} \mathrm{~s}^{-1}, 50$ to 100 times more sensitive than any flown before. This increase in sensitivity was due to larger area, an anticoincidence shield to reduce particle background, and a wide solid angle to increase the probability of observing a source during the flight. The payload shown in Fig. 1 was successful in detecting the first stellar x-ray source in the flight of June 12, 1962 (Giacconi et al., 1962). An individual source (Sco X-1) dominated the night sky and was detected at 28 \pm 1.2 counts $\mathrm{cm}^{-2} \mathrm{~s}^{-1}$, just below the threshold of previous experiments (Fig. 2). No exceptionally bright or conspicuous visible light or radio object was present at that position (which, however, was very poorly known). An early confirmation of our result came from the rocket flight of April 1963 by the NRL group led by Friedman, which also discovered x-ray emission from the Crab Nebula (Bowyer et al., 1964).

The truly extraordinary aspect of the discovery was not that an x-ray star had been found but its extraordinary properties. The $\mathrm{x}$-ray radiation intensity from the Sun is only $10^{-6}$ of its visible light intensity. In Sco X-1, the $\mathrm{x}$-ray luminosity is $10^{3}$ times the visible light inten- sity and it was later determined that the intrinsic luminosity is $10^{3}$ the entire luminosity of the Sun! This was a truly amazing and new type of celestial object. Furthermore, the physical process by which the $\mathrm{x}$ rays were emitted on Sco X-1 had to be different from any process for $\mathrm{x}$-ray generation we knew in the laboratory since it has not been possible on Earth to generate $\mathrm{x}$ rays with

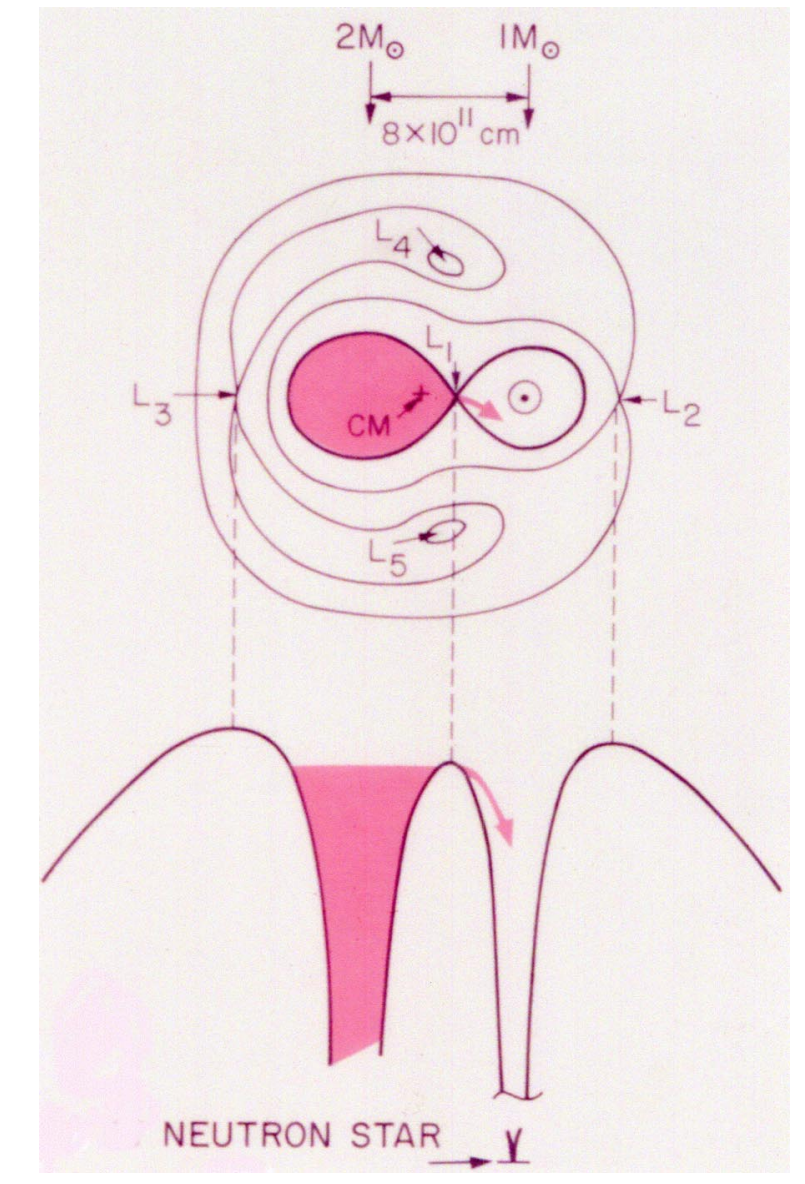

FIG. 9. (Color in online edition) Representation of the equipotentials in the gravitational field of a typical binary x-ray source. Top view and cross section. Illustration of R. Giacconi. 


\section{$99.9 \%$ efficiency.}

Many rocket flights carried out by several groups in the 60 's were able to find new stellar sources and the first extragalactical sources. The NRL group and the Lockheed group (led by Phil Fisher) continued to carry out mostly broad surveys, with the notable exception of the Crab occulation experiment by NRL in 1964 (Bowyer et al., 1964).

The AS\&E group concentrated on the detailed study of individual x-ray sources. Most significant was the series of rocket flights which culminated in the identification of the optical counterpart of Sco X-1. First the group determined that Sco X-1 could not have the thermal spectrum that would be expected from neutron stars (Giacconi et al., 1965), which implied that an optical counterpart should have magnitude 13. In a first rocket flight to measure the angular size of the source it was found to be less than 7 arcsec (Oda et al., 1965). Thus the source had to be a visible star and not a diffused nebulosity. This led to the sophisticated measurement of the location of Sco X-1 by an AS\&E-MIT group led by Herbert Gursky (Gursky et al., 1966), with sufficient precision to enable its identification with a 13thmagnitude star (Sandage et al., 1966) which had spectral characteristics similar to an old nova. This renewed in- terest in a binary star model for Sco X-1 (Burbidge, 1967), and Shklovsky proposed a binary containing a neutron star (Shklovsky, 1967). However, the absence of $\mathrm{x}$-ray emission from other novas, the lack of indications from either the optical spectra or the x-ray data of a binary system, and the general belief that the supernova explosion required to form the neutron star would disrupt the binary system did not lead to the general acceptance of the idea. The discovery by Hewish of pulsars in 1967 turned the attention of the theorists to pulsar models for the x-ray emitters. But such models also were not quite persuasive given the lack of observed $\mathrm{x}$-ray pulsations. The solution to the riddle of Sco X-1 and similar sources was not achieved until the launch of the UHURU satellite, the first of a generation of $\mathrm{x}$-ray observatories.

The proposal to launch a "scanning satellite," which eventually became $U H U R U$, was contained in a document written by Herb Gursky and myself and submitted to NASA on September 25, 1963. In this document we described a complete program of x-ray research culminating in the launch of a 1.2-meter-diameter x-ray telescope in 1968. This youthful dream was not fully realized until the launch of the Chandra X-ray Observatory in 1999, which, not by chance, had a 1.2-meter-diameter

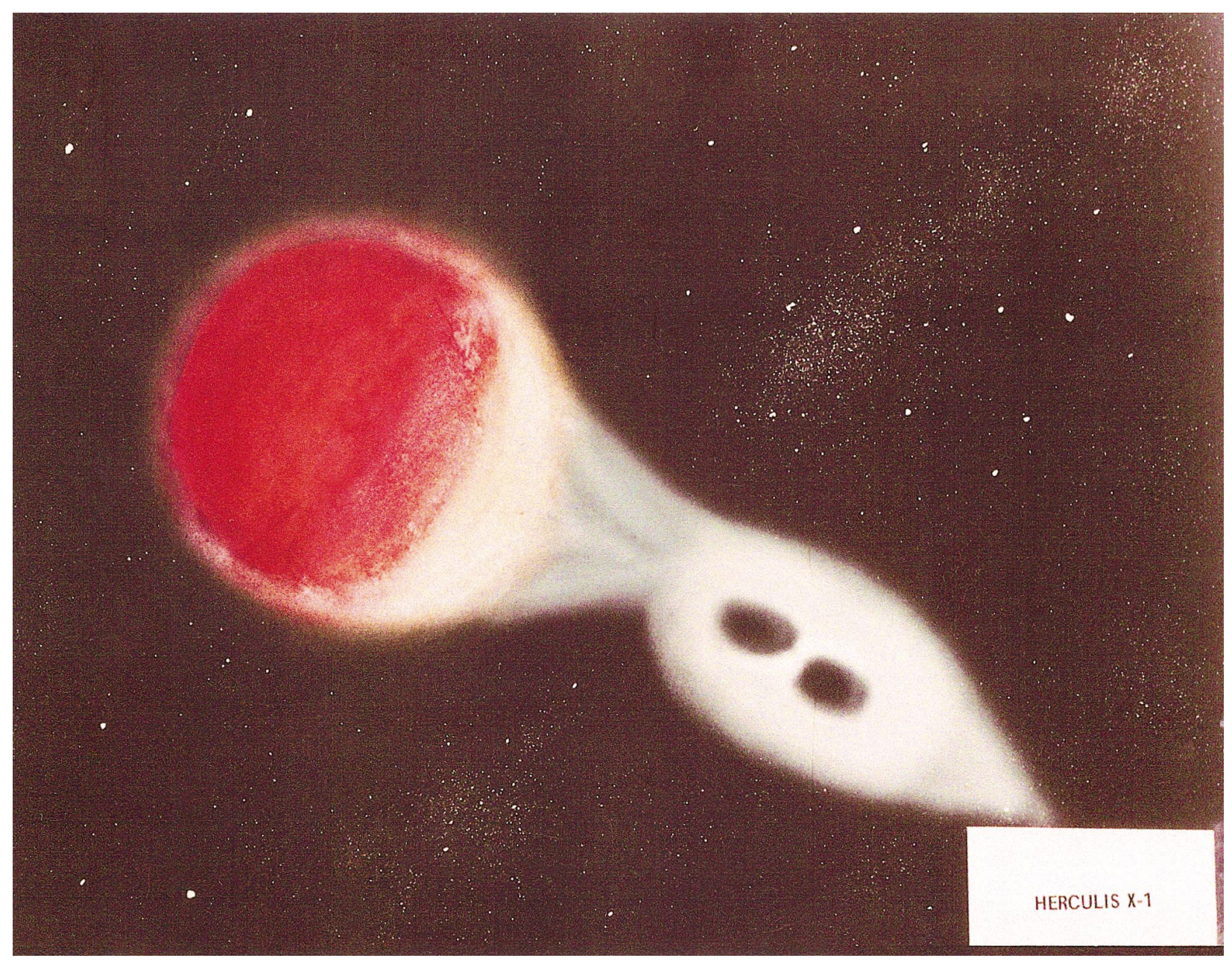

FIG. 10. (Color) An artist's conception of Her X-1 with accretions occurring at the poles of the magnetic field of the neutron star. Illustration of R. Plourde. 


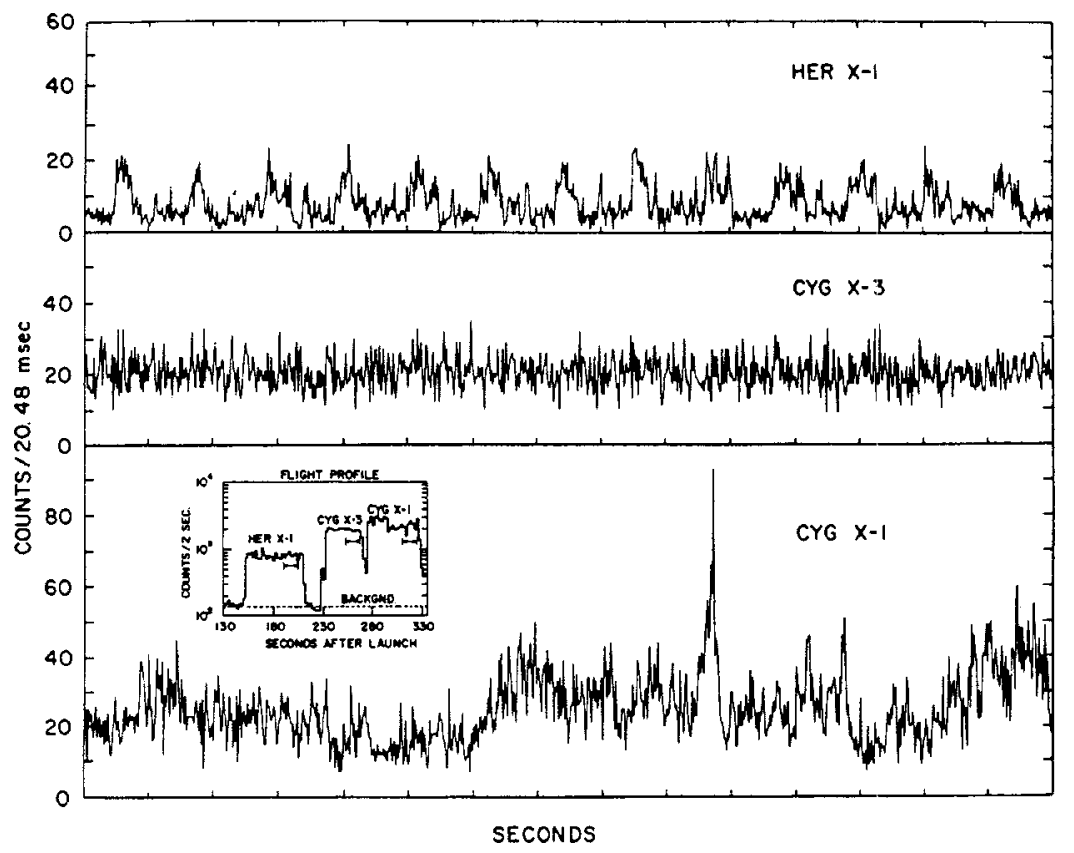

FIG. 11. Comparison of the time variability of Her X-1, Cyg X-3, and Cyg X-1. Courtesy of R. Rothschild.

mirror. But while the difficult technology development that made $\mathrm{x}$-ray telescopes possible was being carried out, the most fundamental advances in x-ray astronomy were made with relatively crude detectors mounted on orbiting satellites.

\subsection{DISCOVERIES WITH UHURU}

The total amount of time which was available for observation of the x-ray sky during the 60's was about one hour: five minutes above $100 \mathrm{~km}$ for each of about a dozen launches. The next step which led us from the

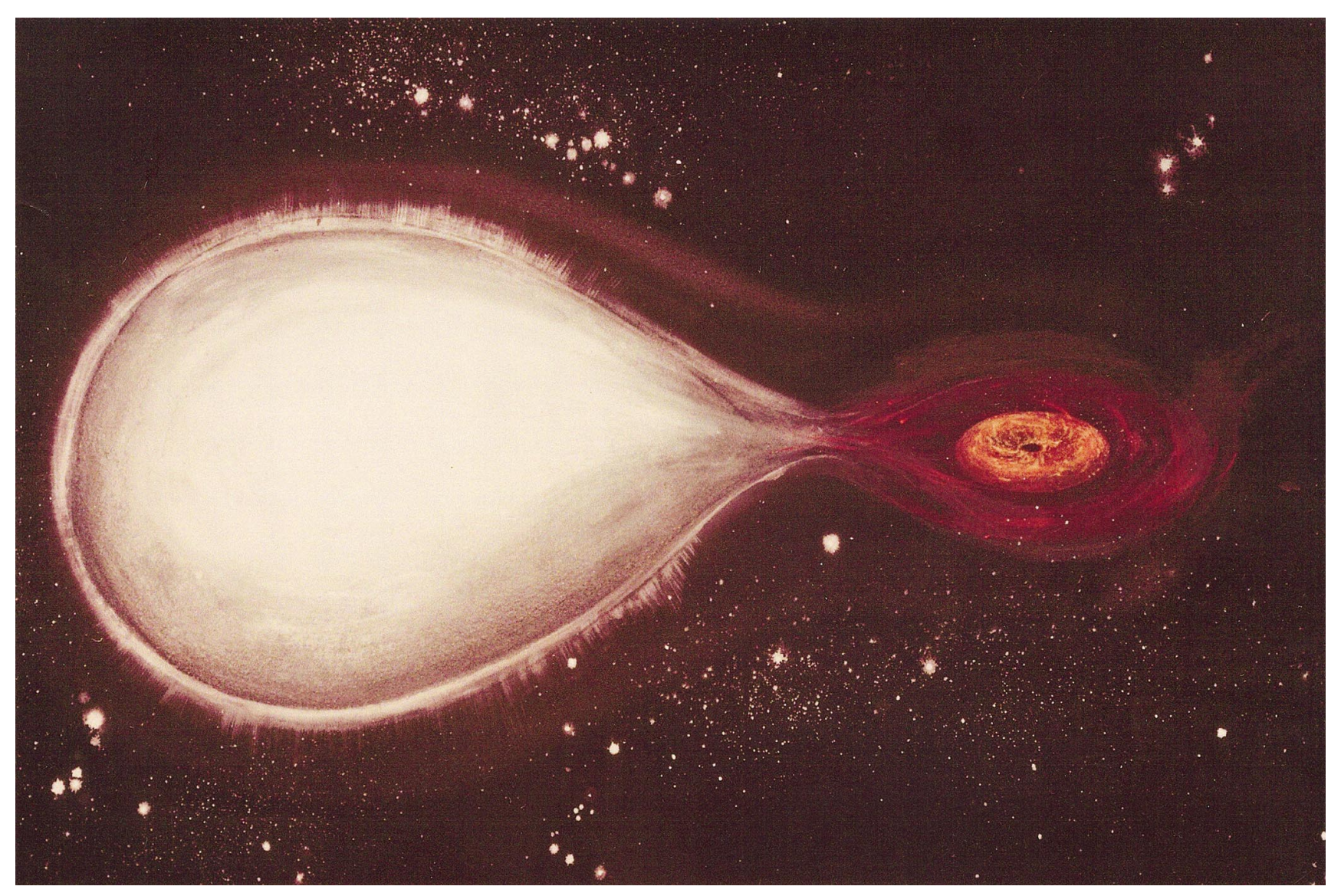

FIG. 12. (Color) Artist's conception of Cyg X-1. Illustration of L. Cohen. 
TABLE II. Consequences of the discovery of binary x-ray systems.

- Existence of binary stellar systems containing a neutron star or a black hole

- Existence of black holes of stellar mass

- Measure of the mass, radius, moment of inertia, and equation of state for neutron stars (Density $10^{15} \mathrm{gr} / \mathrm{cm}^{3}$ )

- A new source of energy due to gravitational infall (100 times more efficient per nucleon than fusion)

- A model (generally accepted) for the nucleus of active galaxies and quasars

phenomenological discoveries to those of great astrophysical relevance occurred on December 12, 1970, when UHURU, the first of the Small Astronomy Satellite series, was launched from the Italian S. Marco platform in Kenya. UHURU was a small satellite (Fig. 3), which we had labored over at AS\&E for seven years between conception, development, testing, and integration.

It was the first observatory entirely dedicated to x-ray astronomy and it extended the time of observation from minutes to years or by five orders of magnitude (Giacconi et al., 1971). The field of view of the detector on board the satellite slowly rotated, examining a $5^{\circ}$ band of the sky that shifted $1^{\circ}$ a day. In three months all the sky could be studied systematically and many new sources could be localized with a precision of about 1 arc min, often permitting the identification of the x-ray sources with a visual or radio counterpart. This in turn led to an evaluation of the distance, the intrinsic luminosity, and the physical characteristics of the celestial object from which the $\mathrm{x}$ rays originated. Among the 300 new sources which were discovered, we were able to identify binary x-ray sources, supernovas, galaxies, active galaxies, quasars, and clusters of galaxies (Fig. 4). But even more important from a certain point of view was the ability, which was provided by the control system, to slow down the satellite spin and spend a very long time on an individual source to study its temporal variations. It was this special ability which permitted the solution of the fundamental unresolved problem of x-ray astronomy until then, namely, the nature of the energy source capable of producing the large intrinsic luminosity of the stellar x-ray sources.

\subsection{The binary $x$-ray sources}

In summary, inspection of the data revealed that some X-ray sources (Her X-1 and Cen X-3; Fig. 5) were regularly pulsating with periods of seconds (Schreier et al., 1972), while others (Cyg X-1) were pulsating with an erratic behavior with characteristic times of less than a tenth of a second, as first noted by Minoru Oda, who was a guest at AS\&E at the time. Ethan Schreier and I noticed that the average intensity of Cen X-3 was modulated over the span of days and that the period of pulsation itself was changing as a function of the phase of the average intensity, which exhibited occulations (Fig. 6). The explanation for this behavior soon became clear:

\section{FOCUSSING X-RAY TELESCOPE}

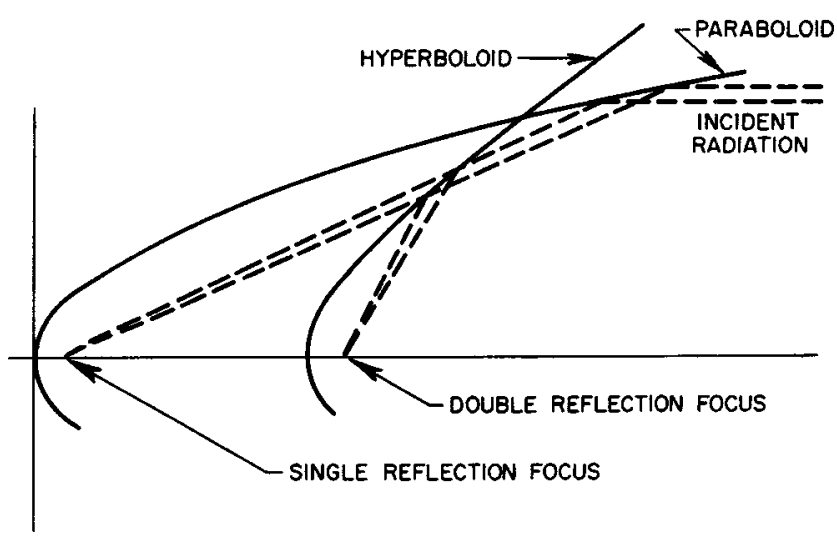

FIG. 13. Principle of x-ray grazing incidence telescope. Illustration of R. Giacconi.

we were observing a stellar x-ray source orbiting a normal star (Fig. 7). The variation of the pulsation period was then due to the Doppler effect. In 1967 Hewish had discovered pulsars in the radio domain. Was this x-ray source a pulsar in orbit about a normal star? This seemed difficult to accept at the time. A pulsar is a neutron star whose formation is believed to be due to the collapse of a star at the end of its life. The concomitant explosion was believed to disrupt any binary system in

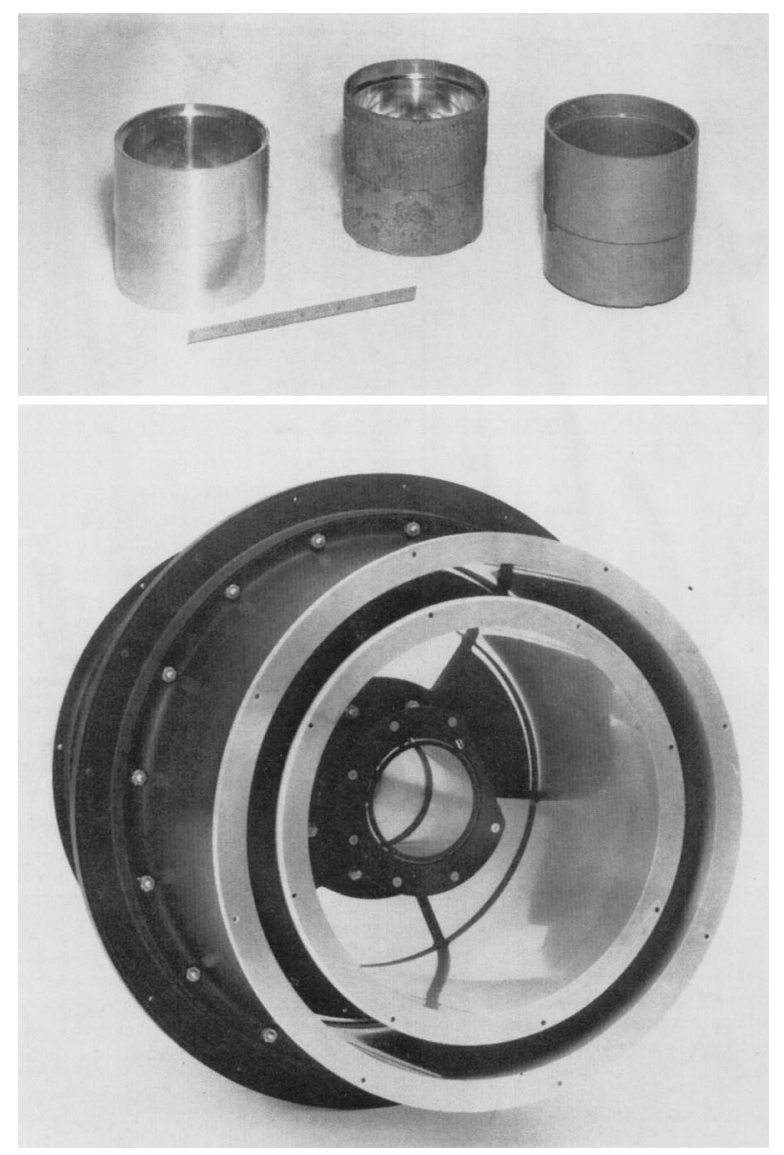

FIG. 14. Several early telescope realizations. From Giacconi, Reidy, et al., 1969. 


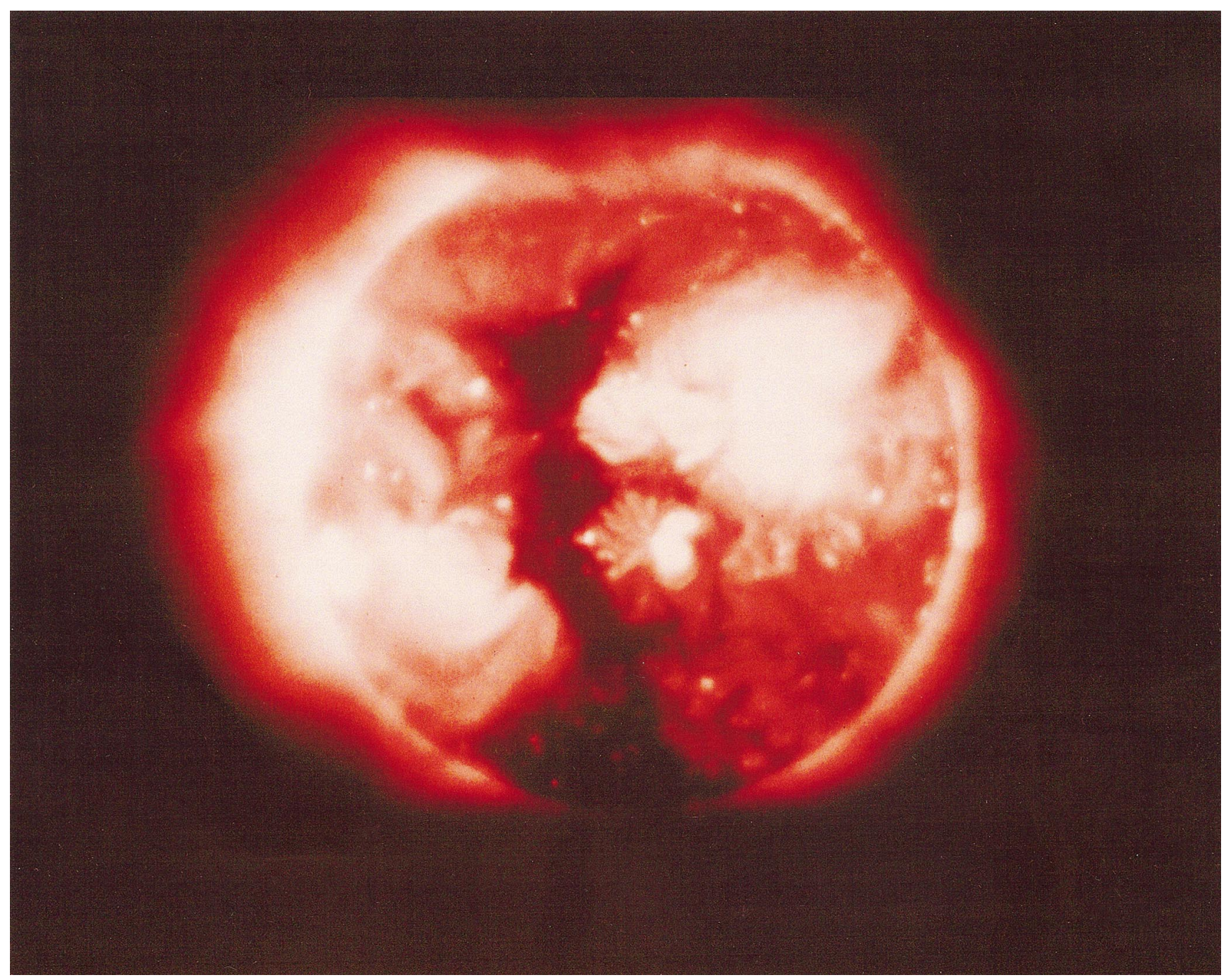

FIG. 15. (Color) Picture of Sun in x rays from Skylab. Courtesy L. Golub, Harvard-Smithsonian Center for Astrophysics.

which it took place. Joseph Taylor had not yet discovered the binary pulsar.

But a new, unexpected, and important finding came to light: the period of pulsation was decreasing rather than increasing with time (Fig. 8). This was true not only in Cen X-3, but also, as Harvey Tananbaum found, in Her X-1 (Tananbaum et al., 1972b). Now this was truly embarrassing! In a pulsar the loss of electromagnetic energy occurs at the expense of the kinetic energy of rotation. But in the $\mathrm{x}$-ray sources the neutron star was acquiring rather than losing energy! The explanation was found in the interaction of the gas in the normal star with the collapsed star. Gas from the outer layer of the atmosphere of the normal star can fall into the strong gravitational field of the collapsed star and acquire energies of order of $0.1 \mathrm{mc}^{2}$ per nucleon. The accelerated nucleons in turn heat a shock of very high temperature above the surface of the neutron star, which emits the observed $x$ rays (Fig. 9). It is this material infall that gives energy to the collapsed object. This is the model for Sco X-1 and most of the galactic x-ray sources. In the case of a neutron star with a strong magnetic field ( $10^{12}$ gauss), the ionized plasma is confined to the poles of the rotating neutron star, generating the observed pe- riodicities (Fig. 10). For a black hole, there exists no surface with particular structures, and therefore the pulsation occurs chaotically (Fig. 11).

The observation by $U H U R U$ of rapid variability in Cyg X-1 reported by Oda (Oda et al., 1971) was soon followed by the rocket flights of the GSFC and MIT groups. These observations clarified that the observed pulsations were not periodic but chaotic (Holt et al., 1971; Rappaport, Doxsey, and Zaumen, 1971). By 1974, the GSFC group had achieved a temporal resolution of 1 millisecond and showed large chaotic fluctuations occurring even on this time scale (Rothschild et al., 1974). Such behavior could be expected to occur if the compact object in the binary system (the $\mathrm{x}$-ray source) was a black hole rather than a neutron star. Stimulated by these findings, the search for optical or radio counterparts had become intense in 1971 and 1972. UHURU had obtained a considerably improved position for Cyg $\mathrm{X}-1$ which was made available to Hjellming and Wade to aid in the search for a radio counterpart (Tananbaum et al., 1971). More refined positions were obtained by the Japanese group led by Oda (Miyamoto et al., 1971) and by the MIT group (Rappaport, Zaumen, and Doxsey, 1971) by use of modulation collimators. Hjellming 


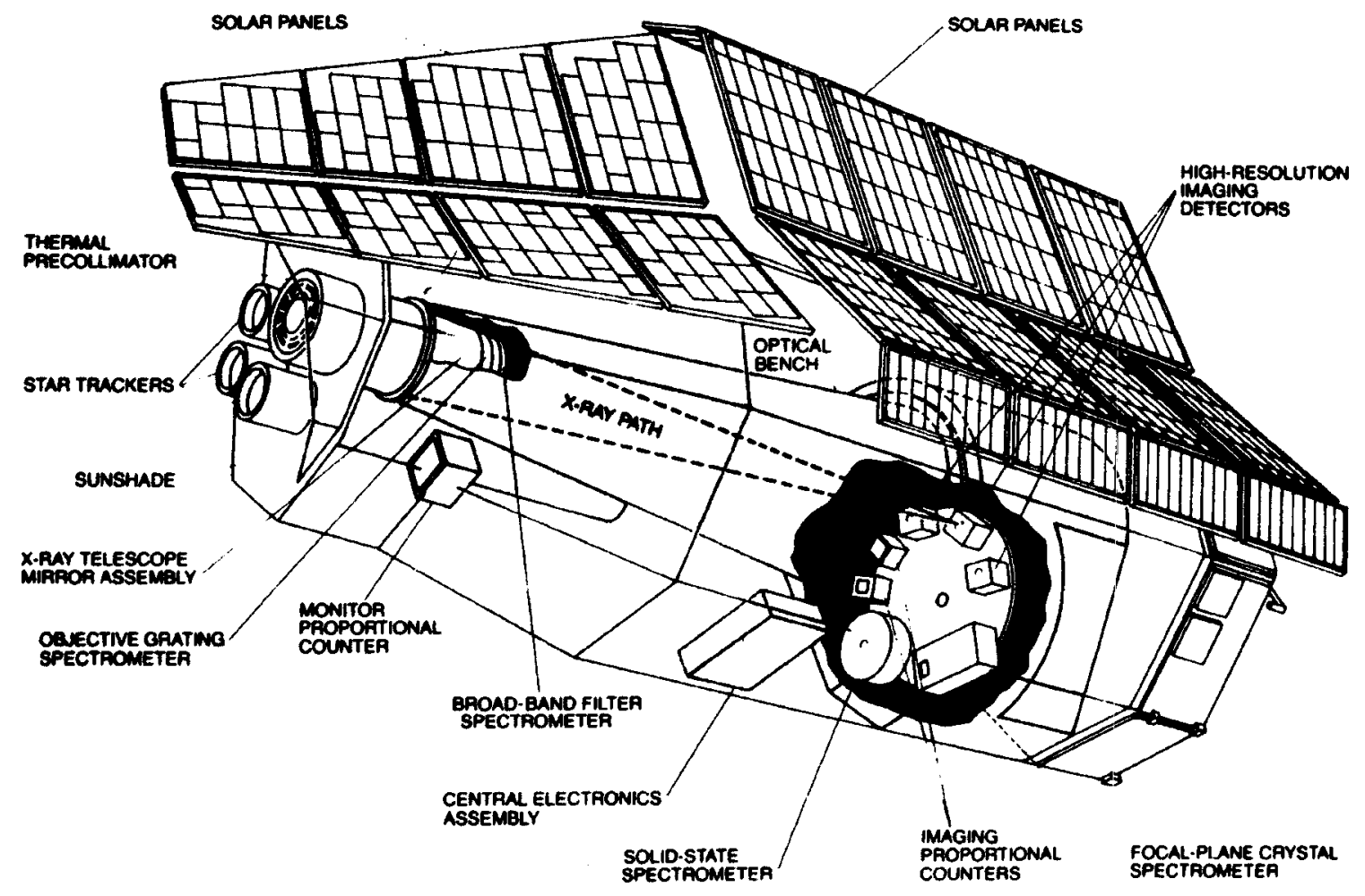

FIG. 16. Schematic representation of the Einstein X-ray Observatory. From Giacconi et al., 1979.

and Wade (1971) and Braes and Miley (1971) reported the discovery of a radio counterpart. The precise radio location led to the optical identification by Webster and Murdin (1972) and by Bolton (1972) of Cyg X-1 with the 5.6-day binary system HDE 226862. The identification of the radio source with Cyg X-1 was confirmed by the observation of a correlated $\mathrm{x}$-ray radio transition in Cyg X-1 (Tananbaum et al., 1972a). Spectroscopic measurements of the velocity of HDE 226862 also permitted Webster and Murdin to establish that Cyg X-1 was indeed in a binary system. The estimated mass for the compact object was greater than six solar masses. Rhoades and Ruffini had shown in 1972 that black holes would have masses greater than 3.4 times the mass of the Sun (Rhoades and Ruffini, 1974).

Thus we could reach conclusions regarding Cyg X-1: the Cyg X-1 x-ray emitter is a compact object of less than $30-\mathrm{km}$ radius due to the rapidity of the pulsations and the fact that the pulsations are so large that they must involve the whole object (Giacconi, 1974). The object has mass greater than that allowed by our current theories for neutron stars. Therefore the object is the first candidate for a black hole (Fig. 12). Currently there are at least six candidates for galactic x-ray sources containing a black hole (Tanaka, 1992).

The consequences of the discovery of binary source $\mathrm{x}$ rays have had far-reaching consequences (Table II). We had proven the existence of binary systems containing a neutron star and of systems containing a black hole. Black holes of solar mass size existed. The binary x-ray sources have become a sort of physical laboratory where we can study the mass, moment of inertia, and equation of state for neutron stars (density $10^{15} \mathrm{gr} / \mathrm{cm}^{3}$ ). We had found a new source of energy for celestial objects: the infall of accreting material in a strong gravitation field. For a neutron star the energy liberated per nucleon is of the order of 50 times greater than that generated in fusion. The above model (of accretion of gas on a collapsed object) has become the standard explanation for the internal engines of quasars and all active nuclei. Recent data seem to confirm the model of accretion on a massive central black hole of $>10^{7}$ solar masses as the common denominator among all the active galaxies.

\subsection{The discovery of high-temperature intergalactic gas}

The establishment of variability in the x-ray universe, discovery of the existence of neutron stars and black holes in binary systems, and the discovery of accretion as a dominant energy source were only the first major

TABLE III. Classes of celestial objects observed with the Einstein Observatory.

- Aurora on Jupiter

- X-ray emission from all types of main-sequence stars

- Novas and supernovas

- Pulsars

- Binary x-ray sources and supernovas in extragalactic sources

- Normal galaxies

- Nuclei of active galaxies

- Quasars

- Groups and clusters of galaxies

- Sources of the extragalactic x-ray background 
accomplishments of $U H U R U$. Among others was a second very important discovery of $U H U R U$ and x-ray astronomy, both because of its intrinsic interest and for its consequences in the field of cosmology, the detection of emission from clusters of galaxies. This emission is not simply due to the sum of the emission from individual galaxies, but originates in a thin gas which pervades the space between galaxies. This gas was heated in the past during the gravitational contraction of the cluster to a temperature of millions of degrees and contains as much mass as that in the galaxies themselves (Gursky et al., 1972). In one stroke the mass of baryons contained in the clusters was more than doubled. This first finding with $U H U R U$, which could detect only the three richest and closest galaxy clusters and with a poor angular resolution of $\frac{1}{2}$ a degree, were followed and enormously expanded by the introduction of a new and powerful x-ray observatory, Einstein, which first utilized a completely new technology in extrasolar $x$-ray astronomy: grazing incidence telescopes.

\subsection{X-RAY TELESCOPES}

Here I must make a short technical diversion to explain the revolution brought about in x-ray astronomy by the telescope technology. When contemplating the estimates made in 1959, I was persuaded that to ultimately succeed in x-ray astronomy, we had to develop new systems quite different from those then in use. Friedman had developed for solar studies a Geiger counter with a thin window which allowed the $\mathrm{x}$ ray to penetrate the interior of the gas volume of the counter. The counter could not decide either the direction of the incoming $\mathrm{x}$ ray or its energy. In order to improve the directional sensitivity, $x$-ray astronomers used collimators, that is, mechanical baffles which defined a field of view typically of $1^{\circ}$. To improve sensitivity we developed anticoincidence shields against spurious particles and enlarged the area. This is what was done in the discovery rocket of 1962. In 1970 UHURU had a very similar detection system. Its improvement in sensitivity was due to

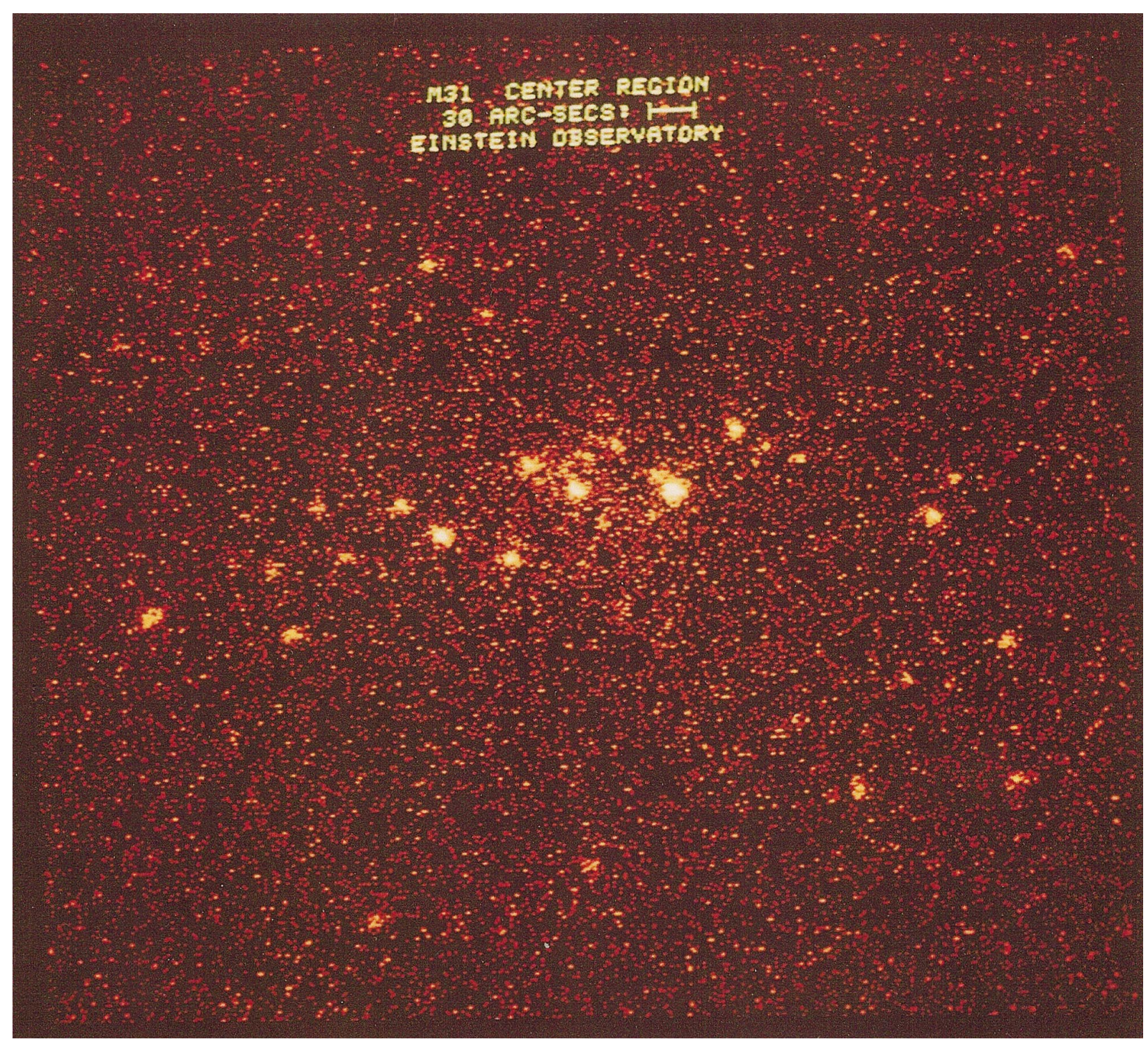

FIG. 17. (Color) X-ray binaries in the galaxy M31. From the Einstein X-ray Observatory. 
the much larger area $\left(800 \mathrm{~cm}^{2}\right.$ instead of 10$)$ and the much longer time of observation. This led to an increase in sensitivity of about $10^{4}$. It should be noted, however, that in the presence of a background noise, the sensitivity improvement was only proportional to the square root of the area. Thus further improvement would have required satellites of football-stadium size. Furthermore, all attempts to gain angular resolution by clever systems of baffles (such as the modulation collimators) led to intrinsically insensitive experiments.

The solution which occurred to me as early as 1959 was to use a telescope just as it is done in visible-light astronomy (Giacconi and Rossi, 1960). This has the great advantage that the flux from a large area of collection is focused onto a small detector, therefore improving both the flux and the signal-to-noise ratio. In addition, high angular resolution can be obtained within a field that is imaged at once, without scanning or dithering motions, therefore yielding an enormous improvement in exposure time for each source in the field.

The only problem is that an x-ray telescope had to be invented, and the technology necessary for its fabrication had to be developed. It ultimately took about 20 years between the conception of the x-ray telescope in 1959 and its first use for stellar x-ray astronomy in 1979. An $\mathrm{x}$-ray telescope is quite different from a visible-light telescope since the wavelength of $\mathrm{x}$-ray photons is comparable to atomic dimensions. According to Lorentz's dispersion theory, it is clear that the index of refraction of $\mathrm{x}$ rays is less than one, which makes optical systems based on refraction essentially impractical, as was realized by Roentgen himself in his classical experiment of 1895. However, x rays can be efficiently externally reflected by mirrors, provided only that the reflection

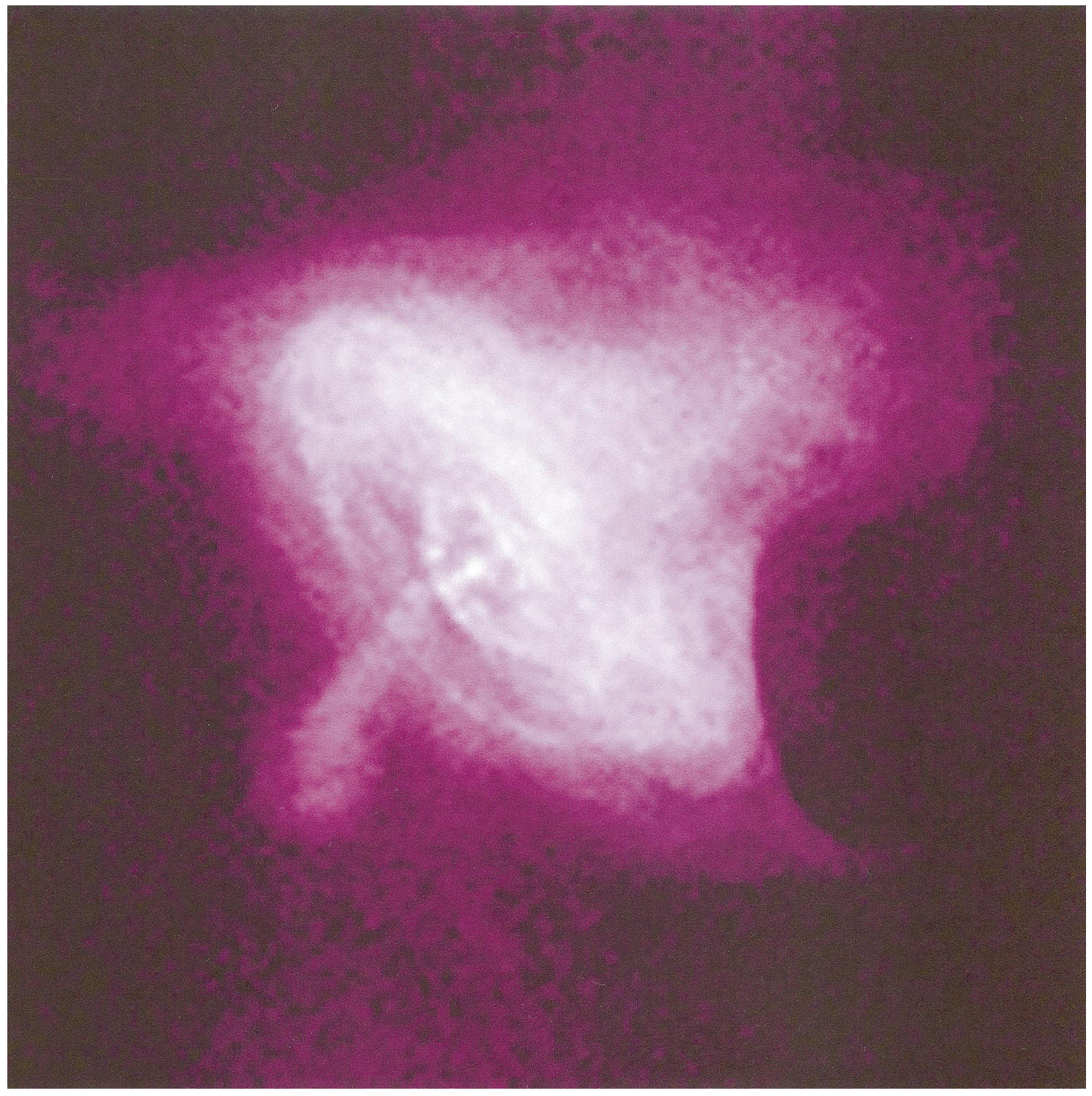

FIG. 18. (Color) The x-ray image of the Crab Nebula obtained from Chandra. Courtesy of NASA. 


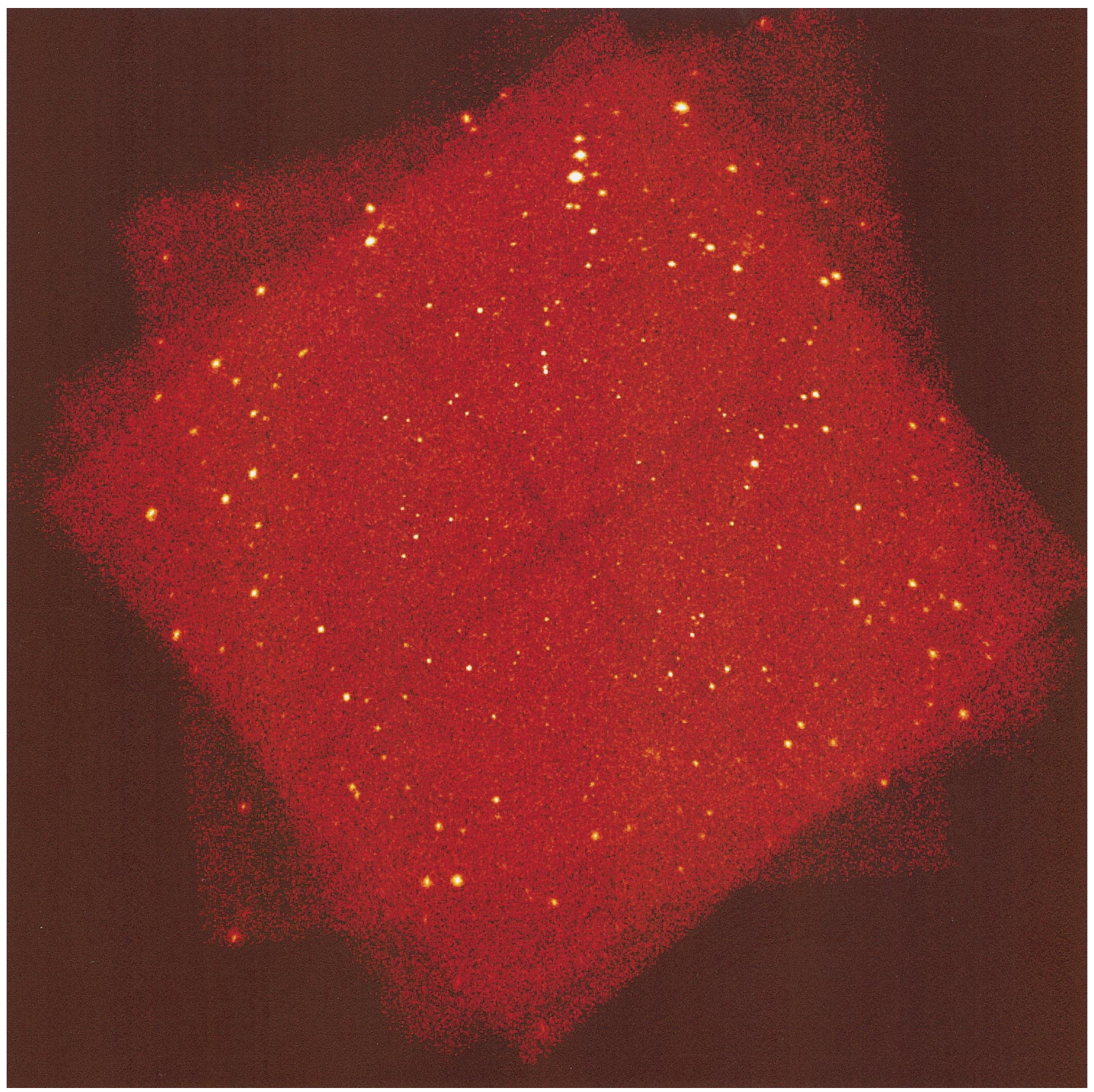

FIG. 19. (Color) The one-million-second exposure in the deep x-ray field from Chandra (CDFS—Chandra Deep Field South).

takes place at very small angles with respect to the mirror's surface. Hans Wolter had already discussed in the 40's and 50's the possibility of using images formed by reflection for microscopy. He showed that using a double reflection from a system of coaxial mirrors consisting of paraboloid and hyperboloid, one could achieve systems with a reasonably large field $\left(1^{\circ}\right)$ corrected for spherical aberrations and coma. Theoretically, therefore, the system was feasible, although the difficulties of construction given the tiny dimension of the systems for microscopy were impossible to overcome (Fig. 13). I persuaded myself, however, that in the corresponding optical designs to be used for telescopes, which required much larger scales (meters rather than microns), such difficulties would not be severe.

In our 1960 paper we described a system that could

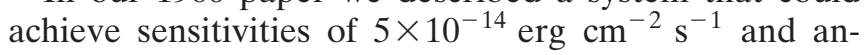

gular resolutions of 2 arc min. The improvement in sensitivity was $10^{6}-10^{7}$ times greater than for any detector then current, and the improvement in angular resolution about a factor of $10^{3}$. Unfortunately it took a long time to develop this technology (Fig. 14; Giacconi et al., 1969). The first primitive pictures of the Sun with an x-ray telescope were obtained in 1965. Giuseppe Vaiana took over leadership of our solar physics program in 1967. In 1973 a high-resolution x-ray telescope studied the Sun over a period of many months with a field large enough to image the disk and nearby corona and with angular resolution finer than 5 arc sec (Fig. 15) (Vaiana and Rosner, 1978).

It was not until 1979 that a fully instrumented x-ray telescope suitable for the detection and study of the much weaker stellar fluxes could be launched. The new satellite, which became known as Einstein, was a real 
astronomical observatory (Fig. 16; Giacconi et al., 1979). In the focal plane of the telescope one could use image detectors with angular resolutions of a few arc sec, comparable to those used in visible light. The sensitivity with respect to point sources was increased by $10^{3}$ with respect to $U H U R U$ and $10^{6}$ with respect to Sco X-1. Spectroscopy could be carried out with a spectral resolving power of 500 .

This substantial technical improvement made possible the detection of all types of astrophysical phenomena (Table III). Auroras due to the Jovian Belts, mainsequence stars of all types, novae, and supernovae were detected. Binary $\mathrm{x}$-ray sources could be studied anywhere in our own galaxy as well as in external galaxies (Fig. 17). Normal galaxies as well as galaxies with active galactic nuclei, such as Seyferts and B Lac, could be detected at very great distances. The most distant quasars ever detected in visible light or radio could be conveniently studied. The sources of the mysterious, isotropic extragalactic background could begin to be resolved.

But to come back to the study of the intergalactic plasma, it is in the study of x-ray emissions from clusters of galaxies that the Einstein observations have had some of the most profound impact. The ability to image the hot plasma has given us the means to study in detail the distribution of the gravitational potential which contains both gas and galaxies. This study could only be carried out with some difficulty by studying individual galaxies, which, even though rather numerous in rich clusters, did not yield sufficient statistical accuracy. The x-ray study has revealed a complex morphology with some clusters exhibiting symmetry and a central maximum of density, which demonstrates an advanced stage of dynamical evolution; but many others show complex structures with two or more maxima. This morphology shows that the merging of the clusters' substructures is not yet completed. The relative youth of many of these clusters had not been sufficiently appreciated previously. The discovery of x-ray emission in clusters is therefore used to study one of the most interesting open questions of modern cosmology, namely, the formation and development of structures in the early epoch of the life of the Universe. Piero Rosati (Rosati et al., 2002) has pushed this work to very distant clusters $(z \sim 1.2)$ by use of the ROSAT Satellite, a splendid successor to Einstein, built at the Max Planck Institute for Extraterrestrial Research by Joachim Trümper and Günther Hasinger and their group.

\subsection{CURRENT RESEARCH WITH CHANDRA}

Harvey Tananbaum and I reproposed the concept of a 1.2-meter-diameter telescope to NASA in 1976. Tananbaum, who had been project scientist on UHURU and scientific program manager on Einstein, gave leadership to the Chandra program and brought it to successful conclusion after I left Harvard in 1981. Chandra has met or exceeded all of our expectations. Comparison of the pictures of the Crab Nebula pulsar by Einstein and by Chandra (Fig. 18) shows the great improvement in sensitivity and angular resolution achieved (Tananbaum and Weisskopf, 2001).

I was able to use Chandra for one million seconds to solve the problem of the sources of the x-ray background, a problem that had remained unsolved since its discovery in 1962 (Fig. 19) (Giacconi et al., 2002). Owing to the great sensitivity and angular resolution of Chan-

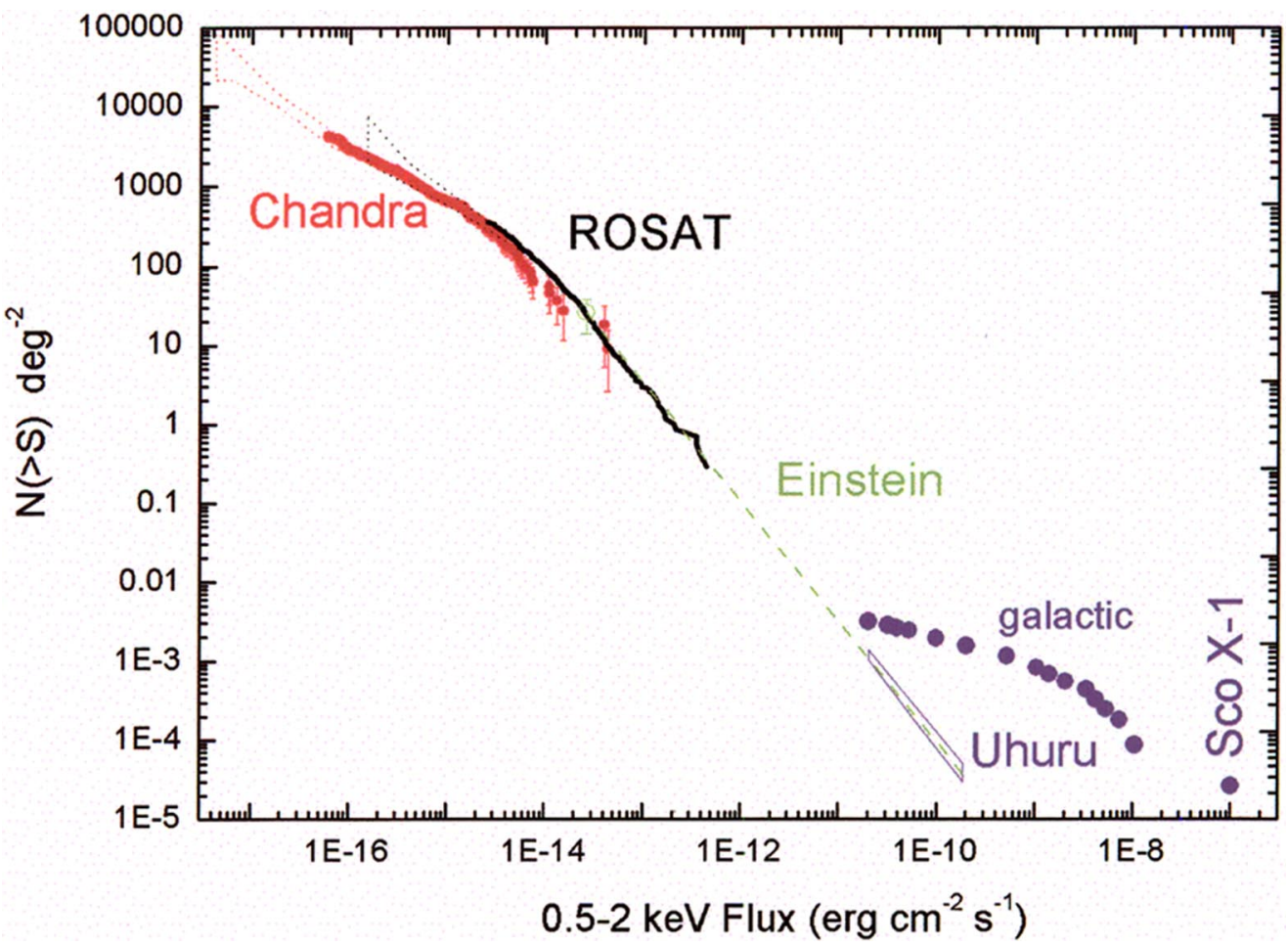

FIG. 20. (Color in online edition) The sensitivity change from 1962 to 2000 (Sco X-1 to Chandra). Courtesy of G. Hasinger. 


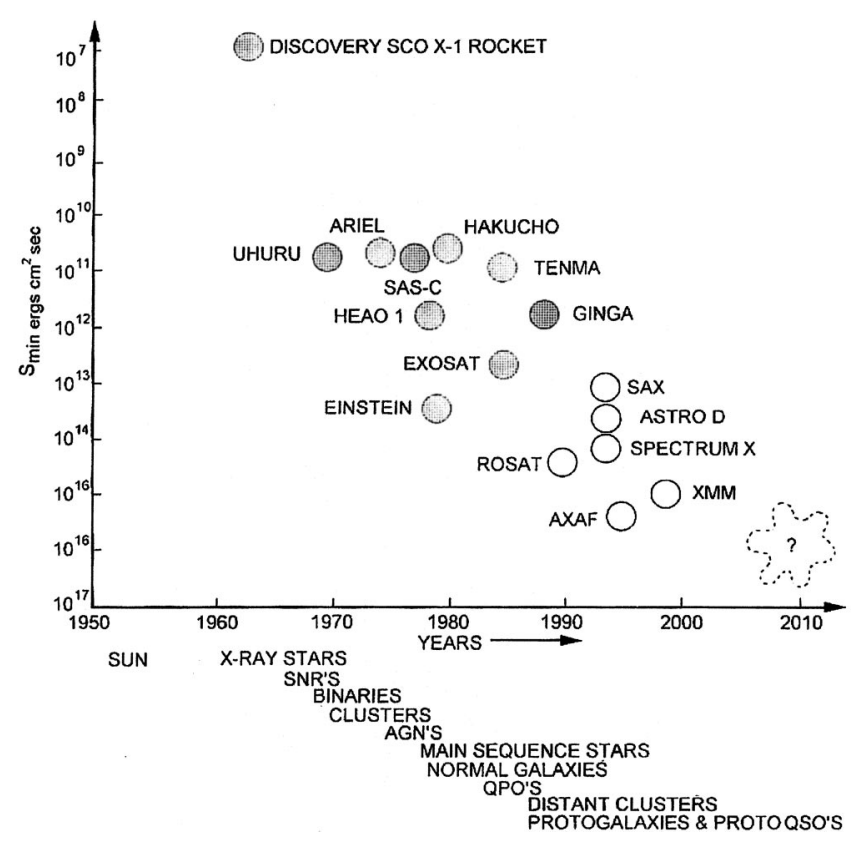

FIG. 21. The sensitivity achieved by various $\mathrm{x}$-ray missions (1970 to 2000). Illustration of R. Giacconi.

$d r a$, we were able to resolve the apparently diffused emission into millions of individual sources. They are active galactic nuclei, quasars, and normal galaxies. The gain in sensitivity that this represents is illustrated in Fig. 20. In Fig. 21, a reminder that x-ray astronomy now involves many groups in the world with distinct and essential contributions.

\subsection{THE FUTURE OF X-RAY ASTRONOMY}

I would like to conclude by attempting to answer the simple question: Why is x-ray astronomy important? The reason is that this radiation reveals the existence of astrophysical processes in which matter has been heated to temperatures of millions of degrees or in which particles have been accelerated to relativistic energies. The $\mathrm{x}$-ray photons are particularly suited to study these processes because they are numerous, because they penetrate cosmological distances, and because they can be focused by special telescopes. This last property significantly distinguishes $x$-ray from $\gamma$-ray astronomy. However, in a more fundamental way, high-energy astronomy has great importance in the study of the Universe, because high-energy phenomena play a crucial role in the dynamics of the Universe.

Gone is the classical conception of the Universe as a serene and majestic ensemble whose slow evolution is regulated by the consumption of nuclear fuel. The Universe we know today is pervaded by the echoes of enormous explosions and rent by abrupt changes of luminosity on large energy scales. From the initial explosion to formation of galaxies and clusters, from the birth to the death of stars, high-energy phenomena are the norm and not the exception in the evolution of the Universe.

\section{REFERENCES}

Bolton, C., 1972, Nature (London) 235, 271.

Bowyer, S., E. T. Byram, T. A. Chubb, and H. Friedman, 1964, Astron. J. 69, 135.

Bowyer, S., E. T. Byram, T. A. Chubb, and H. Friedman, 1964, Science 146, 912.

Braes, L., and G. Miley, 1971, Nature (London) 232, 246.

Burbidge, G. R., 1967, "Theoretical ideas concerning x-ray sources," in Radio Astronomy and the Galactic System: Proceedings IAU Symposium No. 31, edited by Hugo van Woerden (Academic Press, London/New York), p. 463.

Giacconi, R., 1974, "Binary x-ray sources," in Gravitational Radiation and Gravitational Collapse: Proceedings IAU Symposium No. 64, edited by Cecile DeWitt-Morette (Reidel, Dordrecht), p. 147.

Giacconi, R., G. W. Clark, and B. B. Rossi, 1960, "A brief review of experimental and theoretical progress in $\mathrm{x}$-ray astronomy," Technical Note of American Science and Engineering, ASE-TN-49, Jan. 15 (1960).

Giacconi, R., et al., 1979, Astrophys. J. 230, 540.

Giacconi, R., et al., 2002, Astrophys. J., Suppl. Ser. 139, 369.

Giacconi, R., and H. Gursky, 1974, Eds., X-Ray Astronomy, Astrophysics and Space Science Library No. 43 (Reidel, Dordrecht).

Giacconi, R., H. Gursky, F. Paolini, and B. B. Rossi, 1962, Phys. Rev. Lett. 9, 439.

Giacconi, R., H. Gursky, and J. Waters, 1965, Nature (London) 207, 572.

Giacconi, R., E. Kellogg, P. Gorenstein, H. Gursky, and H. Tananbaum, 1971, Astrophys. J. Lett. 165, L27.

Giacconi, R., W. P. Reidy, G. S. Vaiana, L. P. Speybroeck, and T. F. Zehnpfening, 1969, Space Sci. Rev. 9, 3.

Giacconi, R., and B. Rossi, 1960, J. Geophys. Res. 65, 773.

Gursky, H., 1970, in A Survey of Instruments and Experiments for X-ray Astronomy: IAU Symposium No. 37, edited by L. Gratton (Reidel, Dordrecht), p. 19.

Gursky, H., R. Giacconi, P. Gorenstein, J. Waters, M. Oda, H. Bradt, G. Garmire, and B. Sreekantan, 1966, Astrophys. J. 146, 310.

Gursky, H., A. Solinger, E. M. Kellogg, S. Murray, H. Tananbaum, R. Giacconi, and A. Cavaliere, 1972, Astrophys. J. Lett. 173, L99.

Hirsh, R. F., 1979, "Science, Technology and Public Policy: the case of x-ray astronomy, 1959-1972," Ph.D. thesis (University of Wisconsin, Madison).

Hjellming, R. M., and C. M. Wade, 1971, Astrophys. J. Lett. 168, L21.

Holt, S. S., E. A. Boldt, D. A. Schwartz, P. J. Serlemitsos, and R. D. Bleach, 1971, Astrophys. J. Lett. 166, L65.

Miyamoto, S., M. Fujii, M. Matsuoka, J. Nishimura, M. Oda, Y. Ogawara, S. Ohta, and M. Wada, 1971, Astrophys. J. Lett. 168, L11.

Oda, M., G. Clark, G. Garmire, M. Wada, R. Giacconi, H. Gursky, and J. Waters, 1965, Nature (London) 205, 554.

Oda, M., P. Gorenstein, H. Gursky, E. Kellogg, E. Schreier, H. Tananbaum, and R. Giacconi, 1971, Astrophys. J. Lett. 166, L1.

Rappaport, S., R. Doxsey, and W. Zaumen, 1971, Astrophys. J. Lett. 168, L43.

Rappaport, S., W. Zaumen, and R. Doxsey, 1971, Astrophys. J. Lett. 168, L17. 
Rhoades, C., and R. Ruffini, 1974, Phys. Rev. Lett. 32, 324.

Rosati, P., S. Borgani, and C. Norman, 2002, Annu. Rev. Astron. Astrophys. 40, 539.

Rothschild, R., E. A. Boldt, S. S. Holt, and P. J. Serlemitsos, 1974, Astrophys. J. Lett. 189, L13.

Sandage, A., et al., 1966, Astrophys. J. 146, 316.

Shklovsky, I. S., 1967, Astrophys. J. Lett. 148, L1.

Schreier, E., R. Levinson, H. Gursky, E. Kellogg, H. Tananbaum, and R. Giacconi, 1972, Astrophys. J. Lett. 172, L79.

Tanaka, Y., 1992, "Black Hole X-ray Binaries," in Proceedings of Ginga Memorial Symposium: from Ginga to ASTRO-D and further to DUET, ISAS Symposium on Astrophysics, edited by F. Makino and F. Nagase (Institute of Space and Astronautical Science, Tokyo), p. 19.
Tananbaum, H., H. Gursky, E. Kellogg, R. Giacconi, and C. Jones, 1972a, Astrophys. J. Lett. 177, L5.

Tananbaum, H., H. Gursky, E. Kellogg, R. Levinson, E. Schreier, and R. Giacconi, 1972b, Astrophys. J. Lett. 174, L143.

Tananbaum, H., E. Kellogg, H. Gursky, S. Murray, E. Schreier, and R. Giacconi, 1971, Astrophys. J. Lett. 165, L37.

Tananbaum, H., and M. Weisskopf, 2001, in New Century of $X$-ray Astronomy, Astron. Soc. Pacific Conference Proceedings 251, edited by H. Inoue and H. Kunieda (Astron. Soc. Pacific, San Francisco), p. 4.

Vaiana, G. S., and R. Rosner, 1978, Annu. Rev. Astron. Astrophys. 16, 393.

Webster, B., and P. Murdin, 1972, Nature (London) 235, 37. 